\title{
A CHARACTERIZATION OF COCOMPACT HYPERBOLIC AND FINITE-VOLUME HYPERBOLIC GROUPS IN DIMENSION THREE
}

\author{
J. W. CANNON AND DARYL COOPER
}

\begin{abstract}
We show that a cocompact hyperbolic group in dimension 3 is characterized by certain properties of its word metric which depend only on the group structure and not on any action on hyperbolic space. We prove a similar theorem for finite-volume hyperbolic groups in dimension 3.
\end{abstract}

\section{INTRODUCTION}

A group $G$ is called cocompact hyperbolic of dimension $n$ if and only if it can act isometrically, properly discontinuously, and cocompactly on hyperbolic space $\mathbf{H}^{n}$. Proper discontinuity implies that the action has at most finite kernel. Our main result states that a group $G$ is cocompact hyperbolic of dimension 3 if and only if it has a finite generating set $C$ and associated Cayley graph $\Gamma=\Gamma(G, C)$ such that the word metric on $\Gamma$ is comparable in the large (quasiisometric) with the standard hyperbolic metric on $\mathbf{H}^{3}$. We have a similar result for finite volume hyperbolic groups in dimension 3. We believe that our results generalize to all dimensions, at least in the cocompact case. Our result for groups of finite volume employs a different metric space. Instead of the Cayley graph with word metric, we use an augmented Cayley graph associated with a group $G$ and a finite family of Euclidean subgroups of $G$. We prove that the group acts isometrically and properly discontinuously on $\mathbf{H}^{3}$ with orbit space of finite volume if and only if the augmented Cayley graph is quasi-isometric with $\mathbf{H}^{3}$. Precise definitions of all of these terms will appear in $\S 2$. The proof of the cocompact case appears in $\S 3$; the proof of the finite volume case appears in $\S 4$.

Our results are similar to a result of Gromov [G1] but without the added assumption that the group be the fundamental group of a convex path space. There are other results on the classification of groups up to quasi-isometry in [G2]. In particular a group is quasi-isometric to $\mathbf{E}^{n}$ if and only if there is a subgroup $\mathbf{Z}^{n}$ of finite index. It would be interesting to know which groups are quasi-isometric to the 3-dimensional geometries $\mathbf{H}^{2} \times \mathbf{E}^{1}$, Solv, and $\overparen{P S L}(2, \mathbf{R})$. Gromov's work on groups with polynomial growth [G3, BK] implies that a group is quasi-isometric to Nil if and only if it has a subgroup of finite index which is the fundamental group of a compact Nil 3-orbifold. Notice that all compact

Received by the editors November 15, 1988 and, in revised form, January 22, 1990.

1980 Mathematics Subject Classification (1985 Revision). Primary 57M15, 30C60, 30 F40.

The authors were supported in part by grants from the National Science Foundation. 
spaces are quasi-isometric, so that all finite groups are quasi-isometric to $\mathbf{S}^{3}$. Further by the above result of Gromov the groups which are quasi-isometric to $\mathbf{S}^{2} \times \mathbf{E}^{1}$ are precisely those groups containing an infinite cyclic subgroup of finite index.

In [C] Cannon showed how to use the geometry of $\mathbf{H}^{n}$ to give solutions to the word problem and conjugacy problem for cocompact hyperbolic groups. The results were exactly analogous to the original solutions by Dehn for surface groups. Cannon proposes to show in another paper how to use that same hyperbolic geometry, in conjunction with the results of this paper on groups of finite volume, to establish analogous decision algorithms for hyperbolic groups of finite volume.

\section{DEFINITIONS AND PRELIMINARY RESULTS}

Here are the precise definitions. Each metric is a global topological metric rather than an infinitesimal metric. All metrics are denoted by the symbol $d$, perhaps with a subscript to indicate the space. If $A$ is a set and $\varepsilon>0$, then $N(A, \varepsilon)$ denotes the $\varepsilon$-neighborhood of $A$, that is the set of points at distance $<\varepsilon$ from $A$. A group action of $G$ on a metric space $M$ with metric $d_{M}$ is a homomorphism from $G$ into the homeomorphism group $\operatorname{Homeo}(M)$ of $M$. The kernel of this homomorphism is called the kernel of the action. The action is denoted in symbols by $\phi: G \times M \rightarrow M$. The image $\phi(g, x)$ is denoted simply by $g(x)$ or $g x$. A homeomorphism is an isometry if it preserves the distance between points. The action is isometric if the image of $G$ under this homomorphism lies in the subgroup of isometries of the metric space $\left(M, d_{M}\right)$. The action $G \times M \rightarrow M$ is said to be properly discontinuous if, for each compact subset $K$ of $M$, the set $\{g \in G \mid K \cap g(K) \neq \varnothing\}$ is finite. For a properly discontinuous action, it is clear that the kernel of the homomorphism $G \rightarrow \operatorname{Homeo}(M)$ is finite. The set of orbits $M / G$ can be given the identification topology. The resulting space is called the orbit space of the action. The action is called cocompact if the orbit space is compact. In the case where $M$ is hyperbolic space $\mathbf{H}^{n}$ and the action is properly discontinuous, then the orbit space $\mathbf{H}^{n} / G$ inherits a natural volume element from $\mathbf{H}^{n}$. If the total volume of $\mathbf{H}^{n} / G$ is finite, then the action is said to be of finite volume. A group is called finite-volume hyperbolic if it admits an action on $\mathbf{H}^{n}$ that is isometric, properly discontinuous, and of finite volume.

The natural setting for our results is that of geometric group theory which studies a group by means of the geometries on which it acts isometrically. A geometry is a metric space $M$ with path metric $d_{M}$ such that each closed metric ball is compact. A group can act isometrically, properly discontinuously, and cocompactly on some geometry if and only if it is finitely generated. (See [C2].)

If a group $G$ is finitely generated, the Cayley group graph with respect to any finite generating set is a geometry on which the group can act geometrically. Let $G$ denote a group with generating set $C=C^{-1}$. The Cayley group graph $\Gamma=\Gamma(G, C)$ of $G$ with respect to $C$ is a directed graph with vertex set $G$ and with directed edges of the form $e=(v, c, v \cdot c)$, where $v \in G$ and $v \cdot c \in G$ are vertices and $c \in C$ is a generator. The directed edges $e=(v, c, v \cdot c)$ and $e^{-1}=\left(v \cdot c, c^{-1}, v\right)$ represent the same undirected edge of $\Gamma$ and are considered inverses of each other. The group graph just defined has a natural 
path metric called the word metric which assigns to each undirected edge the path length of 1 . The distance between the identity vertex and the vertex $g$ of $\Gamma$ is then the minimal number of edges needed to join the two vertices. This distance is, of course, the minimal word length of a word in the generators $C$ representing the element $g$. The closed metric balls are compact so that $\Gamma$ is a geometry precisely in the case where the generating set is finite. The group $G$ acts isometrically on the group graph $\Gamma=\Gamma(G, C)$ by left multiplication. Left multiplication by $g \in G$ takes the vertex $v \in G$ to the vertex $g \cdot v$ and the edge $e=(v, c, v \cdot c)$ to the edge $(g \cdot v, c, g \cdot v \cdot c)$. If the generating set $C$ is finite, then this isometric action is also properly discontinuous and cocompact.

We call an action geometric if the action is an isometric, properly discontinuous, and cocompact action on a geometry. A finitely generated group can, of course, act geometrically on many different geometries. However, any two geometries on which a finitely generated group acts geometrically are equivalent under an equivalence relation which we shall call quasi-isometry. This relationship is also known as pseudo-isometry. We now explain this relationship. Let $\left(M, d_{M}\right)$ and $\left(N, d_{N}\right)$ be geometries. A relation (= multi-valued function) $R: M \rightarrow N$ is said to be $K$-quasi-Lipschitz, $K \geq 1$, if

(i) $R$ is everywhere defined (i.e., $R(x) \neq \varnothing$ for each $x \in M$ ); and

(ii) for each $A \subset M$, $\operatorname{diam} R(A) \leq K(\operatorname{diam} A+1)$.

That is, the images of small sets must have uniformly bounded diameters, while the images of large sets must have diameters satisfying a Lipschitz condition. "Quasi" (= to some extent), as we use it here, is simply a substitute for "in the large." Relations $R: M \rightarrow N$ and $S: N \rightarrow M$ are $K$-quasi-inverses if each is $K$-quasi-Lipschitz, $d_{M}\left(S \circ R, \mathrm{id}_{M}\right)<K$, and $d_{N}\left(R \circ S, \mathrm{id}_{N}\right)<K$. A $K$-quasiLipschitz relation $R: M \rightarrow N$ is a $K$-quasi-isometry if it has a $K$-quasi-inverse $S: N \rightarrow M$. Given any two quasi-isometries, we may assume, if convenient, that they each are assigned the same parameter $K$; indeed, it suffices to use the larger of given parameters for each. It is an easy exercise to prove that the composition of two $K$-quasi-isometries is a $\left(K^{2}+K\right)$-quasi-isometry.

We recall the following easy and well-known theorem.

Equivalence Theorem. If a group acts geometrically and cocompactly on two geometries $\left(M, d_{M}\right)$ and $\left(N, d_{N}\right)$, then $\left(M, d_{M}\right)$ and $\left(N, d_{N}\right)$ are quasiisometric.

The proof is standard. The reader may construct a proof or consult [C2]. As a start, here are adequate definitions for $R$ and $S$. Fix $\varepsilon>0$. Let $U_{0}$ and $V_{0}$ be bounded, connected open sets in $M$ and $N$, respectively, such that the $G$-translates of $U_{0}$ and $V_{0}$ cover $M$ and $N$, respectively. Let $U$ and $V$ denote the $\varepsilon$-neighborhoods of $U_{0}$ and $V_{0}$, respectively. Define $R: M \rightarrow N$ and $S: N \rightarrow M$ by

$$
\begin{gathered}
R(x)=\{y \in N \mid \exists g \in G, x \in g U, y \in g V\}, \quad \text { and } \\
S(y)=\{x \in M \mid \exists g \in G, x \in g U, y \in g V\} .
\end{gathered}
$$

A notion which plays an essential role in our proofs is that of quasi-geodesic. There are many equivalent formulations. The version that seems easiest to use in our case is the following. Let $I$ denote an interval, finite or infinite, in the space $\mathbf{Z}$ of integers with metric induced from the standard metric on the real 
numbers. Let $M$ denote a metric space with metric $d$. A function $f: I \rightarrow M$ is said to be a $K$-quasi-geoa'esic if for each $A \subset I$ and $B \subset f(I)$

$$
\operatorname{diam} f(A) \leq K(\operatorname{diam} A+1) \quad \text { and } \quad \operatorname{diam} f^{-1}(B) \leq K(\operatorname{diam} B+1) .
$$

That is, $f$ is a $K$-quasi-isometry onto its image, with $f$ chosen to be a function for convenience.

We obtain quasi-geodesics by means of two standard constructions. The first starts with a minimizing geodesic $g:[a, b] \rightarrow M$ from a real interval $[a, b]$, finite or infinite, into a space $M$ with path metric $d_{M}$. One defines $I=\mathbf{Z} \cap[a, b]$ and $f=g \mid I$. Then $f$ is a 1-quasi-geodesic. The second construction starts with a $K$-quasi-geodesic $f: I \rightarrow M$ and a $K$-quasi-isometry $R: M \rightarrow N$ with $K$-quasi-inverse $S: N \rightarrow M$. Let $f^{\prime}: I \rightarrow N$ be any function contained in the composite relation $R \circ f$. An easy argument shows that $f^{\prime}$ is a $K^{\prime}$-quasi-geodesic, with $K^{\prime}=3 K^{2}+K$. Here is a key lemma.

Lemma. Let $\Gamma$ denote a locally finite graph and $\phi: \Gamma \rightarrow \mathbf{H}^{3}$ a K-quasi-isometry. Then there is a constant $W(K)>0$, depending only on $K$, having the following property. Let $x, y \in \mathbf{H}^{3}$. Let $x^{\prime}, y^{\prime} \in \Gamma$ be points such that $d_{H}\left(x, \phi\left(x^{\prime}\right)\right)<K$ and $d_{H}\left(y, \phi\left(y^{\prime}\right)\right)<K$. Let $[x, y]$ denote the hyperbolic geodesic segment joining $x$ and $y$. Let $\alpha\left(x^{\prime}, y^{\prime}\right)$ denote a graph geodesic segment in $\Gamma$ connecting $x^{\prime}$ and $y^{\prime}$. Then $\phi\left(\alpha\left(x^{\prime}, y^{\prime}\right)\right)$ lies in the $W(K)$ neighborhood of $[x, y]$ in $\mathbf{H}^{3}$. Proof. Let $x_{0}, \ldots, x_{n}, x_{0}=x^{\prime}, x_{n}=y^{\prime}$, be points of $\alpha\left(x^{\prime}, y^{\prime}\right)$ appearing in the order named on $\alpha\left(x^{\prime}, y^{\prime}\right)$ with $d_{\Gamma}\left(x_{i-1}, x_{i}\right)=1$ for $i<n$ and $d_{\Gamma}\left(x_{n-1}, x_{n}\right) \leq 1$. Let $f:\left\{x_{0}, \ldots, x_{n}\right\} \rightarrow \mathbf{H}^{3}$ be any function contained in $\phi \mid\left\{x_{0}, \ldots, x_{n}\right\}$. As noted above, $\phi \mid\left\{x_{0}, \ldots, x_{n-1}\right\}$ is a $\left(3 K^{2}+K\right)$-quasigeodesic in $\mathbf{H}^{3}$. By [C, Theorem 2] there is a $W_{0}$ depending only on $3 K^{2}+$ $K$ such that $f\left(\left\{x_{0}, \ldots, x_{n-1}\right\}\right)$ lies in the $W_{0}$ neighborhood of the hyperbolic geodesic segment $\left[f\left(x_{0}\right), f\left(x_{n-1}\right)\right]$ joining $f\left(x_{0}\right)$ and $f\left(x_{n-1}\right)$. But $\left[f\left(x_{0}\right), f\left(x_{n-1}\right)\right]$ lies in the $3 K$ neighborhood of $[x, y]$. Hence we may take $W(K)=W_{0}+3 K$.

Corollary. Let $\Gamma$ denote a locally finite graph and $\phi: \Gamma \rightarrow \mathbf{H}^{3}$ a $K$-quasiisometry. If $\alpha$ is a geodesic line in $\mathbf{H}^{3}$, then there is a geodesic line $A$ in $\Gamma$ such that $\phi(A)$ lies within $W(K)$ of $\alpha$ and $\alpha$ and $\phi(A)$ have the same endpoints at infinity in $\mathbf{S}_{\infty}^{2}$.

Proof. Let $A_{1}, A_{2}, \ldots$ denote geodesic segments in $\Gamma$ such that $\phi\left(A_{1}\right), \phi\left(A_{2}\right)$, ... approximate larger and larger geodesic segments in $\alpha$ as in the lemma. Since each $\phi\left(A_{i}\right)$ lies in the $W(K)$ neighborhood of $\alpha$ and since only finitely many segments of $\Gamma$ have images in the $W(K)$ neighborhood of any given compact segment of $\alpha$, it follows that we may assume that the sequence $A_{1}, A_{2}, \ldots$ converges to a geodesic line $A$ in $\Gamma$ which satisfies the conclusion of the corollary.

\section{COCOMPACT GROUPS}

With the Equivalence Theorem of $\S 2$ in mind, we might well discuss the main theorem once more. A cocompact hyperbolic group acts geometrically on both its Cayley graph and on hyperbolic space. Hence by the Equivalence Theorem a necessary condition that a group be cocompact hyperbolic is that its Cayley 
graph be quasi-isometric with hyperbolic space. Our main theorem shows that this necessary condition is also sufficient.

Theorem. Let $G$ denote a group with finite generating set $C=C^{-1}$. Let $\Gamma=$ $\Gamma(G, C)$ denote the associated Cayley graph. Then $G$ is cocompact hyperbolic in dimension 3 if and only if $\Gamma$ is quasi-isometric with hyperbolic space $\mathbf{H}^{3}$.

Corollary. A group $G$ is the fundamental group of some closed hyperbolic 3manifold if and only if $G$ is finitely generated, torsion free, and the associated Cayley graph is quasi-isometric with $\mathbf{H}^{3}$.

Proof. If $G$ is cocompact hyperbolic in dimension 3 , then, as noted in $\S 1$, the group $G$ is finitely generated and the associated Cayley graph is quasi-isometric with hyperbolic space $\mathbf{H}^{3}$.

Suppose conversely that $G$ has a finite generating set $C=C^{-1}$ with associated Cayley graph $\Gamma=\Gamma(G, C)$ and that $\Gamma$ is quasi-isometric with $\mathbf{H}^{3}$. We need to show that $G$ is cocompact hyperbolic in dimension 3 .

Here is an easy outline of the proof. Let $\phi: \Gamma \rightarrow \mathbf{H}^{3}$ and $\psi: \mathbf{H}^{3} \rightarrow \Gamma$ denote $K$-quasi-inverses. The formula $g x=\phi g \psi(x)$ defines an approximate action of $G$ on $\mathbf{H}^{3}$. The proof then proceeds in five steps. The hard work, outlined in Steps (1), (2), and (3), appears in other papers and is used to show that we may assume that this approximate action extends naturally to the sphere at infinity and that we may assume that the action on the sphere at infinity is conformal:

(1) The approximate action extends to an action of $G$ on the 2 -sphere at infinity. An old argument of $\mathrm{D}$. Mostow [M] shows that the extended action is uniformly quasi-conformal on the 2-sphere.

(2) An argument of $\mathrm{D}$. Sullivan [S] shows that after a quasi-conformal change of coordinates at infinity, the action can be made conformal.

(3) A theorem of W. Thurston [T] or of Tukia and Väisälä [TV] shows that the quasiconformal change of coordinates at infinity may be realized as the map at infinity induced by a bi-Lipschitz homeomorphism of $\mathbf{H}^{3}$. This bi-Lipschitz homeomorphism is of course a quasi-isometry of $\mathbf{H}^{3}$. That is, we may assume that the original action at infinity was conformal.

The conformal action at infinity induces an isometric action on hyperbolic space.

(4) The induced isometric action is properly discontinuous.

(5) The induced isometric action is cocompact.

Steps (4) and (5) require some additional geometric argument which essentially succeeds because the action of $G$ on $\Gamma$ is both properly discontinuous and cocompact, while, up to the factors that matter in the definitions of proper discontinuity and cocompactness, the spaces $\Gamma$ and $\mathbf{H}^{3}$ are equivalent. Now we give the details of the steps.

Step 1 . As noted in $\S 1$, the group $G$ acts isometrically, properly discontinuously, and cocompactly on $\Gamma$ by multiplication on the left. If $\phi$ and $\psi$ are $K$-quasiinverses as indicated above, then we may define an action of $G$ on $\mathbf{H}^{3}$ by the formula $g(x)=\phi(g(\psi(x)))$. Although this action is by relations (multivalued functions) rather than by continuous functions, nevertheless it extends "continuously" in a unique way to the sphere $\mathbf{S}_{\infty}^{2}$ at infinity for $\mathbf{H}^{3}$ and this extension is quasiconformal on $\mathbf{S}_{\infty}^{2}$. Perhaps we should explain the meaning of "continuous" extension in this setting. Let $x_{1}, x_{2}, \ldots$ denote a sequence from 
$\mathbf{H}^{3}$ converging to a point $x$ of $\mathbf{S}_{\infty}^{2}$. Then the sequence of sets $\phi\left(g\left(\psi\left(x_{i}\right)\right)\right)$ converges to a unique point $g(x)$ of $\mathbf{S}_{\infty}^{2}$. This induced action at infinity is called the boundary action. The proof is essentially due to Mostow [M], where he proved that a $K$-quasi-isometry of $\mathbf{H}^{n}$ to itself extends uniquely in the fashion just mentioned to a $K^{2}$-quasiconformal map of $\mathbf{S}^{n-1}$. Though our definitions are slightly different from Mostow's, Mostow's proof is easily adapted to our situation.

Step 2. Now that $G$ acts quasiconformally on $\mathbf{S}_{\infty}^{2}$ with uniform constant of quasi-conformality, a theorem of Sullivan $[\mathrm{S}]$ implies that this action is conjugate, by a quasiconformal map $h: \mathbf{S}_{\infty}^{2} \rightarrow \mathbf{S}_{\infty}^{2}$, to a conformal group action on $\mathbf{S}_{\infty}^{2}$.

Step 3. By a theorem of Thurston [T, Chapter 11] or of Tukia and Väisälä [TV, Theorem 3.2], the map $h$ is the boundary map of a bi-Lipschitz, hence quasiisometric, homeomorphism of $\mathbf{H}^{3}$. Thus, replacing $\phi$ if necessary by $h \circ \phi$, we may assume that our original quasi-isometry $\phi$ induced a conformal action of $G$ on $\mathbf{S}_{\infty}^{2}$. Any conformal action of $G$ on $\mathbf{S}_{\infty}^{2}$ is the boundary action of an isometric action of $G$ on $\mathbf{H}^{3}$. Our original quasi-isometric action of $G$ on $\mathbf{H}^{3}$ was induced by the quasi-isometry $\phi$. We shall denote the new isometric action by $\Phi: G \times \mathbf{H}^{3} \rightarrow \mathbf{H}^{3}$. It remains only to show that this isometric action $\Phi$ of $G$ on $\mathbf{H}^{3}$ is in fact both properly discontinuous and cocompact.

Step 4 . We show that the action $\Phi$ is properly discontinuous.

Fix $O \in \mathbf{H}^{3}$. Let $\alpha$ and $\beta$ denote perpendicular geodesic lines in $\mathbf{H}^{3}$ with intersection $\alpha \cap \beta=\{O\}$. Let $A$ and $B$ denote graph geodesic lines in $\Gamma$ such that $\phi(A)$ shares infinite endpoints with $A$ and $\phi(B)$ shares infinite endpoints with $B$. (See the Corollary from $\S 2$.) Let $C$ denote a graph geodesic segment joining $A$ to $B, C$ of minimal possible length.

There is a positive number $V(K)$, depending only on $K$, such that $\phi(C)$ lies within $V(K)$ of $\{O\}$. Indeed, there exist points $a \in A$ and $b \in B$ such that $\phi(a)$ and $\phi(b)$ lie within $K+W(K)$ of $O$. Hence

$$
d_{\Gamma} \leq K(K+W(K)+1) .
$$

Hence $C$ has length $\leq K(K+W(K)+1)$, and $\phi(C)$ has

$$
\text { diameter } \leq K(\operatorname{diam} C+1) \text {. }
$$

But $\phi(C)$ intersects both the $W(K)$ neighborhood of $\alpha$ and the $W(K)$ neighborhood of $\beta$, and those neighborhoods diverge from one another as one moves away from $O$. The existence of $V(K)$ follows.

Suppose next that $g \in G$. Fix $P>0$ and suppose $g \in G$ satisfies $d_{H}(O, g(O)) \leq P$. We wish to estimate $d_{\Gamma}(C, g C)$. The sets $g(\alpha)$ and $g(\beta)$ are geodesics meeting perpendicularly at $g(\alpha \cap \beta)=g(O), g(A)$ has image $\phi g(A)$ which shares infinite endpoints with $g(\alpha), g(B)$ has image $\phi g(G)$ which shares infinite endpoints with $g(\beta)$, and $g(C)$ is of minimal length joining $g(A)$ and $g(B)$. Hence $\phi g(C)$ lies within $V(K)$ of $g(O)$. Pick $a \in C, b \in g(C)$. Let $a^{\prime} \in \phi(a), b^{\prime} \in \phi(b)$. Let $a^{\prime \prime} \in \psi\left(a^{\prime}\right) \subset \psi \circ \phi(a)$ and 
$b^{\prime \prime} \in \psi\left(b^{\prime}\right) \subset \psi \circ \phi(b)$, where $\psi$ is a $K$-quasi-inverse to $\phi$. Then

$$
\begin{aligned}
d_{\Gamma}(C, g C) & \leq d_{\Gamma}(a, b) \\
& \leq\left[d_{\Gamma}\left(a, a^{\prime \prime}\right)+d_{\Gamma}\left(b, b^{\prime \prime}\right)\right]+d_{\Gamma}\left(a^{\prime \prime}, b^{\prime \prime}\right) \\
& \leq[2 K]+K\left(d_{H}\left(a^{\prime}, b^{\prime}\right)+1\right) \\
& \leq 3 K+K\left[d_{H}\left(a^{\prime}, O\right)+d_{H}\left(b^{\prime}, g(O)\right)+d_{H}(O, g(O))\right] \\
& \leq 3 K+K[2 V(K)+P] .
\end{aligned}
$$

The action of $G$ on $\Gamma$ is properly discontinuous, so that only finitely many elements of $G$ can satisfy $d_{\Gamma}(C, g C) \leq 3 K+K[2 V(K)+P]$. Hence all other elements of $G$ move $O$ a distance $>P$. We conclude that $G$ acts properly discontinuously on $\mathbf{H}^{3}$.

Step 5. Finally we show that the action $\Phi$ of $G$ on $\mathbf{H}^{3}$ is cocompact.

Let $x \in \mathbf{H}^{3}$. Let $x \in \psi(x)$. Let $g \in G$ be a vertex of $\Gamma$ within a distance 1 of $x^{\prime}$. We estimate the distance $d_{H}\left(O, g^{-1} x\right)$ as follows.

$$
\begin{aligned}
d_{H}\left(O, g^{-1} x\right) & =d_{H}(g O, x) \\
& \leq d_{H}(g O, \phi g(C))+d_{H}\left(\phi g(C), \phi\left(x^{\prime}\right)\right)+d_{H}\left(\phi\left(x^{\prime}\right), x\right) .
\end{aligned}
$$

We have

$$
\begin{aligned}
& d_{H}(g O, \phi g(C)) \leq V(K), \\
d_{H}\left(\phi g C, \phi x^{\prime}\right) \leq & K d_{\Gamma}\left(g C, x^{\prime}\right)+K=K\left[d_{\Gamma}\left(C, g^{-1} x^{\prime}\right)\right]+K \\
\leq & K\left(d_{\Gamma}(C, \mathrm{id})+d_{\Gamma}\left(\mathrm{id}, g^{-1} x^{\prime}\right)\right)+K \\
\leq & K\left(d_{\Gamma}(C, \mathrm{id})+K\right)+K, \\
& \quad d\left(\phi x^{\prime}, x\right) \leq K,
\end{aligned}
$$

the last since $\phi x^{\prime} \in \phi \psi(x)$.

We conclude that the closed ball or disk centered at $O$ of radius

$$
V(K)+\left[K\left(d_{\Gamma}(C, \text { id })+K\right)+K\right]+K
$$

contains a representative of each orbit of the action. Hence the action is cocompact.

\section{THE FINITE VOLUME CASE}

The difficulty in the finite volume case is illustrated by the following 2dimensional example. Consider an ideal triangle in hyperbolic 2-space $\mathbf{H}^{2}$. The group $G$ generated by the three reflections in the sides of this triangle acts isometrically and properly discontinuously on $\mathbf{H}^{2}$ with finite 2-dimensional volume (= area). The images of the original triangle tile the hyperbolic plane. But the action is not cocompact. The group $G$ is isomorphic with the free product of three copies of $\mathbf{Z} / 2 \mathbf{Z}$. The graph $\Gamma$ of $G$ can be embedded in $\mathbf{H}^{2}$ : vertices of $\Gamma$ go to an orbit of the action, namely to barycenters of the vertices of the triangles, edges become hyperbolic geodesics joining barycenters of adjacent triangles. If the action were cocompact, then this embedding would be a quasiisometry. However, if one draws the diagram, it is apparent that the embedded graph completely misses infinitely many disjoint circular disks (horoballs) in $\mathbf{H}^{2}$ tangent to the circle at infinity. Each of these disks contains an infinite amount of area of $\mathbf{H}^{2}$. That is, the graph is not really a good approximation to $\mathbf{H}^{2}$. 
In order to obtain a graph that is quasi-isometric with $\mathbf{H}^{2}$, the graph must be augmented by adding certain infinite portions that fill up the omitted horoballs.

In summary, in the finite volume, noncocompact case, we must augment the Cayley graph if we are to obtain a graph quasi-isometric with hyperbolic space. The potential holes in the graph corresponding to omitted horoballs in hyperbolic space are represented by parabolic subgroups of the group. In dimension 3 parabolic subgroups can be easily recognized as groups having an Abelian group of finite index, the Abelian group having rank 2. After one has identified the potential holes and appropriately filled them, then the characterization merges with that of the cocompact case. That is, in dimension 3, a group is finitevolume hyperbolic if and only if its augmented Cayley graph is quasi-isometric with hyperbolic 3-space $\mathbf{H}^{3}$.

Here is a brief outline of this section. The three steps will be carried out in subsections 4.1, 4.2, and 4.3.

(1) We define the augmented Cayley graph.

(2) We show that if a group is finite-volume hyperbolic in dimension 3 , then the augmented Cayley graph is quasi-isometric with hyperbolic 3-space $\mathbf{H}^{3}$.

(3) We show that if the augmented Cayley graph of a group is quasi-isometric with $\mathbf{H}^{3}$, then the group is finite-volume hyperbolic in dimension 3 .

4.1. The augmented Cayley graph. We wish to fill the parabolic holes in a group. We need to be able to recognize those holes. We recall the structure of a finite-volume hyperbolic group $G$ in dimension 3 in order to see how those parabolic holes arise. The structure of $G$ is discussed, for example, in [Ma, Chapter VI]. Here are some standard properties of $G$ and its parabolic subgroups. The group $G$ is finitely generated. Let $x \in S_{\infty}^{2}$ be any point that is fixed by some parabolic element of $G$. Let $G_{x}$ denote the group of all elements of $G$ that fix $x$. Let $P_{x}$ denote the set of all parabolic elements of $G_{x}$ together with the identity element of $G$. Then $P_{x}$ is a free Abelian group of rank 2 . Every Abelian subgroup of $G$ of rank $>1$ lies in some such subgroup $P_{x}$. The group $G_{x}$ is precisely the normalizer of $P_{x}$ in $G$, and the group $G_{x}$ is also its own normalizer in $G$. The group $P_{x}$ is of finite index in $G_{x}$; consequently, the group $G_{x}$ is finitely generated. There are only finitely many subgroups $P_{x}$ or $G_{x}$ of $G$ up to conjugacy. The group $P_{x}$ is called a parabolic subgroup of $G$. Hence we have the following.

Necessary conditions for a group to be finite-volume hyperbolic in dimension 3 . A group $G$ can be finite-volume hyperbolic in dimension 3 only if it satisfies the following conditions. The group $G$ is finitely generated. Any Abelian subgroup of $G$ of rank $>1$ is a subgroup of a maximal Abelian subgroup which has rank 2. We call the maximal Abelian subgroups of $G$ having rank 2 the parabolic subgroups of $G$. Any parabolic subgroup of $G$ has finite index in its normalizer, and the normalizer is its own normalizer. There are only finitely many conjugacy classes of parabolic subgroups of $G$.

Given such a group, we construct the augmented Cayley graph $\Gamma_{+}$as follows.

Let $P_{1}, \ldots, P_{n}$ denote a set of parabolic subgroups of $G$, one from each of the finitely many conjugacy classes. Let $G_{1}, \ldots, G_{n}$ denote the normalizers of the groups $P_{1}, \ldots, P_{n}$. Let $C=C^{-1}$ denote a finite generating set for $G$ which includes, for each $i$, a finite generating set $C_{i}=C_{i}^{-1}$ for each of the 
groups $G_{i}$. We begin with the Cayley graph $\Gamma=\Gamma(G, C)$. We metrize $\Gamma$ with the word metric. This is the graph which we shall augment.

Let $G_{i}$ denote one of the chosen normalizers. For each left coset $g G_{i}$ of $G_{i}$ in $G$, let $\Gamma\left(g G_{i}\right)$ denote the subgraph of $\Gamma$ whose vertices are the vertices of $g G_{i}$ and whose edges are those labelled by the elements of $C_{i}$ which begin and end at points of $g G_{i}$. Consider the graph of the semigroup $g G_{i} \times\{0,1,2, \ldots\}$ with generating set $\left\langle C_{i}, t\right\rangle, t$ generating $\mathbf{Z}_{+}=\{0,1,2, \ldots\}$ additively. Identify $\Gamma\left(g G_{i}\right) \times\{0\}$ with $\Gamma\left(g G_{i}\right)$. The graph $\Gamma$ augmented by the collection of semigroup grapins just described, one for each left coset of each $G_{i}$, is the augmented group graph $\Gamma_{+}$.

One does not assign length 1 to every edge of the augmented group graph. To get an appropriate metric on $\Gamma_{+}$one proceeds as follows. One assigns length 1 to each edge of $g G_{i} \times\{0,1,2, \ldots\}$ labelled by the generator $t$ of $\mathbf{Z}_{+}$. But to any edge in a level $g G_{i} \times\{j\}$ one assigns the length $\exp \{-j\}$. This makes $\Gamma_{+}$a metric space.

Note that the group $G$ acts on the augmented Cayley graph $\Gamma_{+}$by multiplication on the left. This fact has been noted before for the Cayley graph, and the extension to the remainder of the graph is trivial.

\subsection{Quasi-isometry.}

Theorem. Let $G$ denote a finite-volume hyperbolic group, and let $\Gamma_{+}$denote the augmented Cayley graph associated with $G$. Then the graph $\Gamma_{+}$is quasiisometric with hyperbolic space $\mathbf{H}^{3}$.

Proof. We are given that $G$ acts isometrically, properly discontinuously, and with finite-volume on hyperbolic space $\mathbf{H}^{3}$. We describe a quasi-isometry $\phi: \Gamma_{+} \rightarrow \mathbf{H}^{3}$.

We first define $\phi \mid \Gamma$. If $v$ is a vertex of $\Gamma$, then $v \in G$ and we define $\phi(v)=v(0) \in \mathbf{H}^{3}$. If $e=(v, c, v \cdot c)$ is an edge, with $v \in G$ and $c \in C$, then $\phi$ is to take $e$ linearly onto the hyperbolic geodesic segment $[\phi(v), \phi(v \cdot c)]=$ $[v(0), v(c(0))]$ joining $v(0)$ and $v(c(0))$ in $\mathbf{H}^{3}$.

We next define, for each coset $g G_{i}$ of each $G_{i} \in G, \phi \mid \Gamma\left(g G_{i} \times \mathbf{Z}_{+}\right)$. It suffices to define the image of each vertex, for the edges are then to be taken linearly onto the hyperbolic geodesic segments joining the images of the endpoints. Let $p_{i}$ be the point at infinity fixed by the group $G_{i}$. Then the point $g p_{i}$ is the point at infinity fixed by the conjugate group $g \Gamma_{i} g^{-1}$. Fix $v \in G_{i}$. Let $\left[g v(0), g p_{i}\right)$ denote the geodesic ray joining the point $g(v(0))=\phi(g v)$ with the point $g\left(p_{i}\right) \in \mathbf{S}_{\infty}^{2}$ at infinity. Let $x_{0}=g(v(0)), x_{1}, x_{2}, \ldots$ denote points equally spaced on this ray with $d_{H}\left(x_{0}, x_{j}\right)=j$. Define $\phi(g \cdot v, j)=x_{j}$. This completes the definition of $\phi$. It is clear that $\phi$ is $G$-equivariant.

It remains to see that $\phi$ is a quasi-isometry. The conditions that we shall check are those of the following technical lemma whose proof we shall omit. We remark only that the proof is essentially the same as the proof of $[\mathrm{C}$, Theorem 1] or of the Equivalence Theorem of $\S 1$. (Recall that $N(A, \varepsilon)$ denotes the $\varepsilon$ neighborhood of the set $A$.)

Lemma. Let $X$ and $Y$ be spaces with path metrics $d_{X}$ and $d_{Y}$. In order that a function $\phi: X \rightarrow Y$ be a quasi-isometry it is sufficient that

(i) for some $L>0, Y \subset N(\phi(X), L)$;

(ii) for some $K>0$ and for all $x_{1}, x_{2} \in X, d_{Y}\left(\phi\left(x_{1}\right), \phi\left(x_{2}\right)\right) \leq K$. $d_{X}\left(x_{1}, x_{2}\right)$; and 
(iii) for each $M>0$ there exists an $N>0$ such that if $d_{X}\left(x_{1}, x_{2}\right)>N$ then $d_{Y}\left(\phi\left(x_{1}\right), \phi\left(x_{2}\right)\right)>M$.

We now verify the three conditions of the lemma. It is helpful first to study the structure of $\mathbf{H}^{3}$ relative to $G$.

The group $G$ has a fundamental domain $D$ with finitely many sides. Let $p_{1}, \ldots, p_{n}$ be the points fixed by the groups $G_{1}, \ldots, G_{n}$ as above. Each of these points is a translate of one of the cusps on the domain D. Let $N_{1}, \ldots, N_{n}$ denote disjoint open horoball neighborhoods of $p_{1}, \ldots, p_{n}$ in $\mathbf{H}^{3}$. Require that the $G$ translates of the $N_{i}$ fill up precisely a family of disjoint open horoballs in $\mathbf{H}^{3}$. The neighborhoods $N_{i}$ may be chosen so small that

$$
\phi(\Gamma) \subset \mathbf{H}^{3}-\bigcup\left\{g N_{i}: g \in G ; i=1, \ldots, n\right\} .
$$

We call $Q=\mathbf{H}^{3}-\bigcup\left\{g N_{i}: g \in G ; i=1, \ldots, n\right\}$ the cocompact part of $\mathbf{H}^{3}(\operatorname{rel} G)$. This set $Q$ has a compact intersection with the fundamental domain D. This decomposition of $\mathbf{H}^{3}(\operatorname{rel} G)$ into a compact and a horoball part can be altered in an inessential way by adjusting the sizes of the $N_{i}$.

We now check condition (i) of the lemma. Let $x \in \mathbf{H}^{3}$. Then either there exists an $i, 1 \leq i \leq n$, and $g \in G$ such that $x \in g N_{i}$ or there exists $g \in G$ such that $x \in g\left(\mathbf{D}-\bigcup N_{i}\right)$. In the latter case,

$$
d_{H}\left(x, \phi \Gamma_{+}\right) \leq \operatorname{Diam} g\left(\mathbf{D}-\bigcup N_{i}\right)<\infty .
$$

In the former case, let $B$ denote the horoball $g N_{i}$. A geodesic arc of length $\leq 1$ takes $x$ toward the center $p=g p_{i}$ of the horoball $B$ and onto a horosphere $S$ centered at $p$ which contains the $\phi$-image of the vertices of some level $G_{p} \times\{j\}$ of the subgroup $G_{p}$ of $G$ fixing $p$. Every point of $S$ is a uniformly small distance $\delta$ from a vertex of $G_{p} \times\{j\}$, the size $\delta$ being independent of $i$ and $S$. This observation completes the proof that condition (i) of the lemma is satisfied with $L \geq \max \left\{\right.$ Diam $\left.g\left(\mathbf{D}-\bigcup N_{i}\right), \delta+1\right\}$.

We now check condition (ii). Every edge of $\Gamma_{+}$has either a label $c \in C$ or a label $t$ or a label $t^{-1}$. Each edge with label $t$ or $t^{-1}$ is mapped isometrically by $\phi$. An edge of $\Gamma_{+}$with label $c$ lies in a level $\Gamma\left(G_{p} \times\{j\}\right)$ and has length $1 / e^{j}$. The horospherical distance between the image endpoints is $\left(1 / e^{j}\right)$ times the horospherical distance between the image endpoints of an edge in $\Gamma$ with label $c$. The ratio between hyperbolic distance and horospherical distance is uniformly bounded since the hyperbolic distance is uniformly bounded. It follows that the image edges have lengths whose ratios to preimage lengths are uniformly bounded. Condition (ii) follows immediately.

To check condition (iii) we assume $M>0$ given. The neighborhoods $N_{i}$ of the cusps may be chosen so small that the only portion of $\Gamma_{+}$mapped into a horoball $B_{p}$ centered at $p$ and formed from translates of $N_{i}$ lies in the associated augmentation $\Gamma\left(G_{p} \times \mathbf{Z}_{+}\right)$. We then consider the expansion of the cocompact part $Q$ to the closed hyperbolic $(M+1)$-neighborhood $\operatorname{cl}(N(Q, M+1))$ of $Q$ in $\mathbf{H}^{3}$. Now let $x_{1}, x_{2}, \in \Gamma_{+}$be given such that $d_{H}\left(\phi\left(x_{1}\right), \phi\left(x_{2}\right)\right) \leq M$. There are two cases to consider.

Case 1. Suppose $\phi\left(x_{1}\right)$ and $\phi\left(x_{2}\right)$ lie in $\operatorname{cl}(N(Q, M+1))$. If $\Gamma_{0}$ denotes the portion of $\Gamma_{+}$mapped into $\operatorname{cl}(N(Q, M+1))$ by $\phi$, then $\phi \mid \Gamma_{0}: \Gamma_{0} \rightarrow$ $\operatorname{cl}(N(Q, M+1))$ is a $G$-equivariant map between two spaces on which $G$ acts 
cocompactly. Hence this map is a quasi-isometry. It follows that there is a constant $M^{\prime}$ depending only on $M$ and not on $x_{1}$ and $x_{2}$ such that the distance between $x_{1}$ and $x_{2}$ in $\operatorname{cl}(N(Q, M+1))$ is less than or equal to $M^{\prime}$. The $M^{\prime}$ neighborhood of a compact fundamental domain for

$$
G \mid \operatorname{cl}(N(Q, M+1)), \quad \operatorname{cl}(N(Q, M+1))
$$

having its path metric, has compact closure. Hence the preimage of such a neighborhood has finite diameter $D$ in $\Gamma_{+}$, this diameter depending only on the fundamental domain and $M^{\prime}$ and not on $x_{1}$ and $x_{2}$. Hence $d\left(x_{1}, x_{2}\right)$ is uniformly bounded by $D$, independent of $x_{1}$ and $x_{2}$.

Case 2. Suppose $\phi\left(x_{1}\right)$ and $\phi\left(x_{2}\right)$ do not lie in $\operatorname{cl}(N(Q, N+1))$. Then $\phi\left(x_{1}\right)$ and $\phi\left(x_{2}\right)$ lie in a single horoball $B_{p}$ as described above. The radial distance (toward $p$ ) between levels of $\phi\left(x_{1}\right)$ and $\phi\left(x_{2}\right)$ is bounded by $M$. The horospherical distance between projections into a single level near $\phi\left(x_{1}\right)$ and $\phi\left(x_{2}\right)$ is a bounded multiple of $M$. The horospherical graph distance is a bounded multiple of horospherical distance. Hence graph distance is a bounded multiple of $M$.

Cases 1 and 2 complete the procf of (iii). Hence $\phi$ is a quasi-isometry.

\subsection{Recognizing a finite-volume group.}

Theorem. Let $G$ denote a group satisfying the necessary conditions of subsection 4.1. Let $\Gamma_{+}$denote the augmented Cayley graph associated with $G$. If the graph $\Gamma_{+}$is quasi-isometric with hyperbolic space $\mathbf{H}^{3}$, then the group $G$ is finite-volume hyperbolic in dimension 3.

Proof. The proof proceeds in five steps, exactly as in the cocompact case.

Step 1. There are no changes from the cocompact case. Again, Mostow's argument applies. Hence there is an induced quasi-conformal action at infinity.

Step 2. There are no changes from the cocompact case. Again, Sullivan's argument applies. Hence after a quasi-conformal change of coordinates we may assume that the action at infinity is conformal.

Step 3. There are no changes from the cocompact case. Again, Thurston's or Tukia-Väisälä's argument applies. The quasi-conformal change of coordinates at infinity may be realized by a bi-Lipschitz homeomorphism in $\mathbf{H}^{3}$. Hence we lose no generality in assuming that the original action at infinity was conformal.

Step 4. There are no changes from the cocompact case. Again we conclude that the induced isometric action on $\mathbf{H}^{3}$ is properly discontinuous. In particular, the kernel of the action is finite. It follows that the parabolic groups of $G$ have images under the action that are truly parabolic in $\mathbf{H}^{3}$.

Step 5. It remains only to show that the action is of finite-volume. To that end we want to alter the quasi-isometry $\phi$ so that it more nearly approximates the function defined in the proof of the theorem in subsection 4.2 . We need the following lemma.

Lemma (the action of a parabolic quasi-isometry on horoballs). Suppose that $h: \mathbf{H}^{3} \rightarrow \mathbf{H}^{3}$ is a $K$-quasi-isometry, that $h_{\infty}$ is the quasiconformal extension of $h$ to infinity, and that $h_{\infty}$ is conformal and parabolic with fixed point $x$ at 
infinity. Then $h$ preserves horoballs at $x$ within a fixed bound depending only on $K$.

Proof of the horoball lemma. We may take as model for $\mathbf{H}^{3}$ the Poincare upper half space with $x$ at infinity. A point $p$ on a horoball centered at $x$ is determined by the vertical ray $R$ through $p$ and by any geodesic $S$ through $p$ orthogonal to $R$. The transformation $h_{\infty}$ is then a Euclidean isometry of the Euclidean plane $\mathbf{S}_{\infty}^{2} \backslash\{x\}$ which extends to a natural Euclidean isometry of the upper half space model of $\mathbf{H}^{3}$ which we also denote by $h_{\infty}$, preserves horoballs at $x$, takes vertical rays to vertical rays, and preserves the Euclidean shape and size of all other geodesics. In particular the images of $R$ and $S$ under $h_{\infty}$ intersect at the $h_{\infty}$ image of $p$. As noted earlier, the transformation $h$ preserves geodesics within a uniform bound depending only on $K$. It follows that the image $h(p)$ of $p$ lies within a uniform bound of $h_{\infty}(p)$, the bound depending only on $K$.

We are now ready to complete the proof of the theorem. Fix one of the parabolic subgroups $G_{i}$ of $G$. When we augmented the group graph along $G_{i}$, we added a family of parallel rays with edges labelled by the letter $t$. All of these rays are graph geodesics, hence their images in $\mathbf{H}^{3}$ converge to points at infinity. Since the rays get closer and closer to each other toward infinity, they converge to the same point $x$ at infinity. Each of the transformations of $G_{i}$ fixes $x$. Since $G_{i}$ has an Abelian subgroup of rank 2, and since the kernel of the action is finite, the image of $G_{i}$ is a group of conformal transformations fixing $x$ and having an Abelian subgroup of rank 2 of finite index. Each of the elements of the image must either be parabolic or elliptic, the parabolics forming a subgroup of finite index. Of course, the elements of $G$ do not act directly on $\mathbf{H}^{3}$, but we lose no generality in assuming that $\phi$ embeds $\Gamma_{+}$on which $G$ does act faithfully. It is easy to extend the action discontinuously but quasi-isometrically to all of $\mathbf{H}^{3}$ by mapping each point of $\mathbf{H}^{3}$ to a nearby point of the image of $\phi$. The lemma then applies and shows that the augmentation part of the graph is $K$-dense in some horoball at $x$. In particular the remainder of the graph cannot really intrude on that horoball after a certain point. What that allows one to do is to make the mapping $\phi$ truly $G$ equivariant on the horoball near $x$ without losing the property of quasi-isometry. One can change the map in exactly the same way in each $G$ translate of that horoball, and one can deal similarly with the other groups $G_{j}$.

With $\phi$ altered as in the preceding paragraph, one can return to the proof of $\S 3$ which showed the cocompactness of the group $G$ of $\S 3$. The same argument shows that the $G$ of $\S 4$ acts cocompactly off of the set of horoballs just dealt with. But there are only finitely many $G$ orbits of such horoballs, and the quotient of each has finite volume. We conclude that $G$ is of finite volume.

\section{REFERENCES}

[BK] P. Buser and H. Karcher, Gromov's almost flat manifolds, Astérisque 81 (1981).

[C1] J. W. Cannon, The combinatorial structure of cocompact discrete hyperbolic groups, Geom. Dedicata 16 (1984), 123-148.

[C2] _ The theory of negatively curved spaces and groups, Ergodic Theory, Symbolic Dynamics and Hyperbolic Spaces (Tim Bedford, Michael Keane and Caroline Series, eds.), Oxford Univ. Press, Oxford, New York and Tokyo, 1991, pp. 315-369. 
[F] W. J. Floyd, Group completions and limit sets of Kleinian groups, Invent. Math. 57 (1980), 205-218.

[G1] M. Gromov, Hyperbolic manifolds, Groups and Actions, Proc. Stony Brook Conf., Ann. of Math. Studies No. 97, Princeton Univ. Press, Princeton, N.J., 1981, pp. 183-215.

[G2] _ Infinite groups as geometric objects, Proc. Internat. Congr. Math., Elsevier, New York, 1983, pp. 385-392.

[G3] _ Groups of polynomial growth and expanding maps, Inst. Hautes Études Sci. Publ. Math. 53 (1981), 53-73.

[Ma] Bernard Maskit, Kleinian groups, Springer-Verlag, Berlin, Heidelberg and New York, 1988.

[M] G. D. Mostow, Strong rigidity of locally symmetric spaces, Ann. of Math. Studies No. 78, Princeton Univ. Press, N.J., 1972.

[S] D. Sullivan, On the ergodic theory at infinity of an arbitrary discrete group of hyperbolic motions, Proc. Stony Brook Conf., Ann. of Math. Studies No. 97, Princeton Univ. Press, Princeton, N.J., 1981, pp. 465-496.

[T] W. P. Thurston, The geometry and topology of 3-manifolds, Lecture Notes, Princeton Univ., 1978.

[TV] P. Tukia and J. Väisälä, Quasiconformal extension from dimension $n$ to $n+1$, Ann. of Math. (2) 115 (1982), 331-348.

Department of Mathematics, Brigham Young University, Provo, Utah 84602

Department of MATHEMATICS, WARWick University, Coventry CV4 7AL, ENGLAND Current address: Department of Mathematics, University of California at Santa Barbara, Santa Barbara, California 93107 